\title{
Características clínicas de pacientes con falla cardiaca aguda según la función ventricular izquierda
}

\author{
Paola Calvachi Prieto ${ }^{a, b}$, Juan Sebastián Almánzar ${ }^{a}$, María Paula Vega ${ }^{a}$, \\ Yenny Rocío Cárdenas ${ }^{\mathrm{b}}$, Mabel Gómez ${ }^{\mathrm{c}}$, Edgar Celis ${ }^{\mathrm{b}}$ y Andrés Felipe Buitrago ${ }^{\mathrm{b}, \mathrm{d}, *}$
}

\author{
a Universidad de Los Andes, Facultad de Medicina, Bogotá, Colombia \\ b Departamento de Medicina Crítica y Cuidado Intensivo, Hospital Universitario Fundación Santa Fe de Bogotá, Bogotá, Colombia \\ c Hospital Universitario Fundación Santa Fe de Bogotá, Bogotá, Colombia \\ d Departamento de Medicina Interna, sección de Cardiología, Hospital Universitario Fundación Santa Fe de Bogotá, Bogotá, \\ Colombia
}

Recibido el 8 de abril de 2017; aceptado el 10 de junio de 2017

Disponible en Internet el 12 de octubre de 2017

\section{PALABRAS CLAVE \\ Falla cardiaca aguda; Fracción de eyección del ventrículo izquierdo; Enfermedad coronaria}

\begin{abstract}
Resumen
Introducción: la insuficiencia cardiaca aguda es la causa más frecuente de hospitalización en pacientes mayores de 65 años. A la fecha se han publicado pocos estudios descriptivos en la población colombiana, por lo que se realizó un estudio observacional que expone las características clínicas de los pacientes con diagnóstico de falla cardiaca aguda en Colombia.

Métodos: se hizo un estudio observacional tipo corte-transversal con información obtenida de historias clínicas de pacientes con diagnóstico de falla cardiaca aguda entre los años 2011 a 2015 en la Fundación Santa Fe de Bogotá. Estos datos se recolectaron a través del Registro Colombiano de Enfermedad Cardiovascular (RECODEC).

Resultados: se analizaron los datos de 550 pacientes, con un promedio de edad de 77 años. El análisis univariado evidenció mayor prevalencia de la enfermedad en hombres (55\%) y una importante relación con hipertensión arterial $(78,2 \%)$, enfermedad coronaria $(31,8 \%)$ y fibrilación auricular $(29,6)$. En cuanto a la estancia hospitalaria, se observó un promedio de 8 días en hospitalización general y de 1 día en la unidad de cuidado intensivo. En el análisis bivariado se dividieron los pacientes en tres grupos según la fracción de eyección del ventrículo izquierdo: disminuida $(<40 \%)$, limítrofe $(41-49 \%)$ y preservada $(>50)$. Como desenlaces finales se evaluaron mortalidad $(8,4 \%)$ y aparición de arritmias $(22,5 \%)$.
\end{abstract}

\footnotetext{
* Autor para correspondencia.

Correo electrónico: abuitrag@uniandes.edu.co (A.F. Buitrago).
} 
Conclusiones: el análisis de la base de datos de RECODEC se ha utilizado para evaluar los aspectos epidemiológicos y clínicos de la enfermedad cardiovascular. Existe la necesidad de crear registros nacionales que en general permitan analizar el comportamiento de la población colombiana.

(C) 2017 Sociedad Colombiana de Cardiología y Cirugía Cardiovascular. Publicado por Elsevier España, S.L.U. Este es un artículo Open Access bajo la licencia CC BY-NC-ND (http:// creativecommons.org/licenses/by-nc-nd/4.0/).

\section{KEYWORDS}

Acute heart failure;

Left ventricle

ejection fraction;

Coronary heart

disease

\section{Clinical characteristics of acute heart failure patients according to left ventricular function}

\begin{abstract}
Introduction: Acute heart failure is the most frequent cause of hospital admissions in patients over 65 years-old. Only few descriptive studies have been published on the Colombian population. The aim is to carry out an observational study that determines the clinical characteristics of patients with acute heart failure in a hospital in Colombia.

Methods: An observational, cross-sectional study was conducted using information from clinical records of patients diagnosed with acute heart failure between 2011 and 2015 in our Foundation. These data were collected using the Colombian Registry of Cardiovascular Disease (RECODEC). Results: The analysis was performed on the data from 550 patients, with a median age of 77 years. The univariate analysis showed a higher prevalence of the disease in men (55\%), and a significant association with hypertension $(78.2 \%)$, coronary disease $(31.8 \%)$, and atrial fibrillation $(29.6 \%)$. In the bivariate analysis, the patients were divided into three groups according to their left ventricular ejection fraction: decreased (<40\%), restricted $(41 \%-49 \%)$, and preserved ( $>50 \%$ ), with the preserved ejection fraction being the most prevalent. The main outcomes were mortality $(8.4 \%)$ and the occurrence of arrhythmias $(22.5 \%)$.

Conclusions: It is important to take into account that the analysis of the RECODEC database has been used to evaluate the epidemiological and clinical aspects of cardiovascular disease. However, there is a need to create registries at a national level in order to analyse the general characteristics of the Colombian population.

(C) 2017 Sociedad Colombiana de Cardiología y Cirugía Cardiovascular. Published by Elsevier España, S.L.U. This is an open access article under the CC BY-NC-ND license (http:// creativecommons.org/licenses/by-nc-nd/4.0/).
\end{abstract}

\section{INTRODUCCIÓN}

En las últimas décadas, la falla cardiaca aguda se ha convertido en un importante problema de salud pública, alcanzando una prevalencia que oscila entre el 1 y el $2 \%$ de la población adulta en países desarrollados ${ }^{1}$. Se estima que esta patología es el motivo más frecuente de hospitalización en pacientes mayores de 65 años, siendo la primera causa de mortalidad en Europa y Norteamérica ${ }^{2}$. Aunque en Colombia se cuenta con el registro elaborado por el Ministerio de Salud (MINSALUD), las estadísticas para esta patología son escasas, hecho que dificulta la realización de estudios locales que permitan elaborar políticas de promoción de la salud y prevención de la enfermedad. En el último año en Colombia, la tasa de mortalidad de pacientes con falla cardiaca fue de 6,4 por 100.000 habitantes, siendo la tercera causa de muerte en el país ${ }^{3}$.

La insuficiencia cardiaca es un síndrome clínico caracterizado por anormalidades funcionales o alteraciones estructurales que intervienen en el llenado o la eyección ventricular. Entre los factores de riesgo están la enfermedad coronaria, la hipertensión, la diabetes, y/o la enfermedad valvular ${ }^{4}$. Asimismo, se asocia con falla renal, enfermedad pulmonar obstructiva crónica (EPOC), edad avanzada y sexo masculino ${ }^{3}$. Gran parte de los estudios existentes a la fecha realizan descripciones de población caucásica en países desarrollados, y dejan en un segundo plano los datos de pacientes latinoamericanos. Uno de los principales estudios nacionales, fue desarrollado por Serrano y Hernández en Bucaramanga, en el que se incluyeron 218 pacientes con diagnóstico de falla cardiaca y factores de riesgo como hipertensión arterial $73,1 \%$, seguida de enfermedad coronaria $17 \%$, diabetes mellitus tipo $221,6 \%$ y dislipidemia $11,9 \%{ }^{5}$. Debido a esto y a la falta de información local, se realizó un estudio observacional en la Fundación Santa Fe de Bogotá con el objetivo de presentar su epidemiología y analizar parte de los resultados de la base de datos RECODEC (Registro Colombiano de Enfermedades Cardiovasculares) entre los años 2011 a 2015.

\section{Métodos}

Se llevó a cabo un estudio observacional de tipo cortetransversal con la información obtenida de las historias clínicas de pacientes con diagnóstico de falla cardiaca entre los años 2011 a 2015 en la Fundación Santa Fe de Bogotá. 
Tabla 1 Antecedentes en pacientes con falla cardiaca aguda

\begin{tabular}{|c|c|c|c|}
\hline Variables $(n=550)$ & & Frecuencia & Porcentajes \\
\hline Tabaquismo & $\mathrm{Si}$ & 217 & 39,5 \\
\hline $\begin{array}{l}\text { Fibrilación } \\
\text { auricular }\end{array}$ & $\mathrm{Si}$ & 175 & 31,8 \\
\hline $\begin{array}{l}\text { Enfermedad } \\
\text { cardiovascular }\end{array}$ & $\mathrm{Si}$ & 211 & 38,4 \\
\hline $\begin{array}{c}\text { Enfermedad } \\
\text { vascular } \\
\text { periférica }\end{array}$ & $\mathrm{Si}$ & 48 & 8,7 \\
\hline Diabetes & $\mathrm{Si}$ & 157 & 28,5 \\
\hline Hipertensión & $\mathrm{Si}$ & 430 & 78,2 \\
\hline $\begin{array}{l}\text { Enfermedad } \\
\text { hepática }\end{array}$ & $\mathrm{Si}$ & 12 & 2,2 \\
\hline EPOC & $\mathrm{Si}$ & 121 & 22,0 \\
\hline $\begin{array}{l}\text { Enfermedad } \\
\text { tiroidea }\end{array}$ & $\mathrm{Si}$ & 163 & 29,6 \\
\hline Infarto previo & $\mathrm{Si}$ & 163 & 29,6 \\
\hline $\begin{array}{l}\text { Revascularización } \\
\text { miocárdica }\end{array}$ & $\mathrm{Si}$ & 89 & 16,2 \\
\hline $\begin{array}{l}\text { Enfermedad renal } \\
\text { crónica }\end{array}$ & $\mathrm{Si}$ & 104 & 18,9 \\
\hline Quimioterapia & No & 535 & 97,3 \\
\hline $\begin{array}{l}\text { Ataque } \\
\text { cerebrovascular }\end{array}$ & No & 477 & 86,7 \\
\hline Depresión & No & 527 & 95,8 \\
\hline Alcohol & No & 507 & 96,2 \\
\hline
\end{tabular}

Los datos se obtuvieron a través de RECODEC, que cuenta con variables demográficas, farmacológicas, antecedentes patológicos y exámenes paraclínicos.

El análisis de datos se hizo a través del programa estadístico SPSS versión 23 y Epidat 3.1, obteniéndose frecuencias y porcentajes para las variables cualitativas, y medidas de tendencia central y de dispersión para las cuantitativas; el análisis bivariado se realizó por sexo, fracción de eyección del ventrículo izquierdo (FEVI) (disminuida, limítrofe, conservada) y FEVI (disminuida y conservada).

El muestreo fue no probabilístico por conveniencia de acuerdo con los criterios de inclusión y exclusión.
Tabla 2 Etiología de la falla cardiaca aguda

\begin{tabular}{|c|c|c|}
\hline Variables $(n=550)$ & Frecuencia (n) & Porcentajes (\%) \\
\hline \multicolumn{3}{|c|}{ Etiología falla cardiaca } \\
\hline Isquémica & 235 & 47,8 \\
\hline Valvular & 95 & 19,3 \\
\hline Restrictiva & 9 & 1,8 \\
\hline Hipertrófica & 25 & 5,1 \\
\hline Dilatada & 58 & 11,8 \\
\hline Chagásica & 7 & 1,4 \\
\hline Hipertensiva & 42 & 8,5 \\
\hline Otras* & 21 & 4,3 \\
\hline
\end{tabular}

*En la categoría de otras se tienen en cuenta enfermedades como miocardiopatía arritmogénica del ventrículo derecho y Takotsubo.

\section{Resultados}

Se analizó un total de 550 pacientes; solo dos variables presentaron datos perdidos: alcoholismo y etiología de la falla cardiaca ( $4 \%$ y 10,5\% respectivamente). En el análisis univariado se evidenció mayor porcentaje de hombres (55\%); el promedio de edad fue 77 años con un rango intercuartil de 1,7. En cuanto al tipo de afiliación al sistema general de seguridad social en Colombia, $283(51,5 \%)$ pacientes contaban con medicina prepagada, $173(31,5 \%)$ con entidades promotoras de salud (EPS), 66 (12\%) estaban adscritas al régimen especial en salud, que en el país hace referencia a militares o empresas petroleras, y $28(5,1 \%)$ pacientes no tenían afiliación alguna. Para las variables cuantitativas, el promedio de estancia hospitalaria en pisos general fue de 8 días, con un rango intercuartil de 11, y para los días de permanencia en la unidad de cuidado intensivo ( $\mathrm{UCI}$ ) fue de 1 día con un rango intercuartil de 5 .

Los antecedentes en pacientes con falla cardiaca, se muestran en la tabla 1. Los datos estadísticos de los antecedentes clínicos, farmacológicos y de abuso de sustancias, reflejan que el $78,2 \%$ de los pacientes con falla cardiaca tienen diagnóstico de hipertensión arterial, 38,4\% enfermedad coronaria, 31,8\% fibrilación auricular, 29,6\% hipotiroidismo, $28,5 \%$ diabetes, $22 \%$ EPOC y $18,9 \%$ enfermedad renal crónica. Los principales motivos de consulta fueron disnea en

Tabla 3 Etiología de la falla cardiaca aguda según fracción de eyección

\begin{tabular}{|c|c|c|c|c|c|c|c|}
\hline & \multicolumn{6}{|c|}{ FEVI 3} & \multirow[t]{2}{*}{ Tota } \\
\hline & $<40 \%$ & $\%$ & $40-49 \%$ & $\%$ & $>50 \%$ & $\%$ & \\
\hline \multicolumn{8}{|c|}{ Etiología falla cardiaca } \\
\hline Isquémica & 76 & 32,3 & 68 & 28,9 & 91 & 38,8 & 235 \\
\hline Valvular & 13 & 13,7 & 13 & 13,7 & 69 & 72,6 & 95 \\
\hline Restrictiva & 4 & 44,4 & 1 & 11,1 & 4 & 44,4 & 9 \\
\hline Hipertrófica & 5 & 20 & 2 & 8 & 18 & 72 & 25 \\
\hline Dilatada & 26 & 44,8 & 11 & 19 & 21 & 36,2 & 58 \\
\hline Chagásica & 5 & 71,4 & 1 & 14,3 & 1 & 14,3 & 7 \\
\hline Hipertensiva & 8 & 19 & 6 & 14,3 & 28 & 66,7 & 42 \\
\hline Otras* & 2 & 9,5 & 2 & 9,5 & 17 & 81 & 21 \\
\hline
\end{tabular}

* En la categoría de otras se tienen en cuenta enfermedades como miocardiopatía arritmogénica del ventrículo derecho y Takotsubo. 
Tabla 4 Antecedentes en pacientes con falla cardiaca aguda según fracción de eyección

\begin{tabular}{|c|c|c|c|c|c|c|c|}
\hline & \multirow[b]{2}{*}{$<40 \%$} & \multirow[b]{2}{*}{$\%$} & \multicolumn{3}{|c|}{ FEVI 3} & \multirow[b]{2}{*}{$\%$} & \multirow[t]{2}{*}{ Total } \\
\hline & & & $40-49 \%$ & $\%$ & $>50 \%$ & & \\
\hline \multicolumn{8}{|l|}{ Sexo } \\
\hline Hombre & 97 & 31,8 & 72 & 23,6 & 136 & 44,6 & 305 \\
\hline \multicolumn{8}{|l|}{ Seguro médico } \\
\hline Ninguno & 6 & 21,4 & 11 & 39,3 & 11 & 39,3 & 28 \\
\hline EPS & 58 & 33,5 & 36 & 20,8 & 79 & 45,7 & 173 \\
\hline Prepagada & 70 & 24,7 & 54 & 19,1 & 159 & 56,2 & 283 \\
\hline Régimen especial & 18 & 27,3 & 9 & 13,6 & 39 & 59,1 & 66 \\
\hline \multicolumn{8}{|l|}{ Cigarrillo } \\
\hline $\mathrm{Si}$ & 64 & 29,5 & 43 & 19,8 & 110 & 50,7 & 217 \\
\hline \multicolumn{8}{|l|}{ Fibrilación auricular } \\
\hline $\begin{array}{l}\text { Si } \\
\text { Enfermedad cardiovascular }\end{array}$ & 50 & 28,5 & 33 & 18,9 & 92 & 52,6 & 175 \\
\hline $\mathrm{Si}$ & 77 & 36,5 & 52 & 24,6 & 82 & 38,9 & 211 \\
\hline \multicolumn{8}{|l|}{ Ataque cerebrovascular } \\
\hline $\mathrm{Si}$ & 20 & 27,4 & 14 & 19,2 & 39 & 53.4 & 73 \\
\hline \multicolumn{8}{|l|}{ Depresión } \\
\hline $\mathrm{Si}$ & 8 & 34,8 & 4 & 17,4 & 11 & 47,8 & 23 \\
\hline \multicolumn{8}{|l|}{ Diabetes } \\
\hline $\mathrm{Si}$ & 47 & 29,9 & 31 & 19,8 & 79 & 50,3 & 157 \\
\hline \multicolumn{8}{|l|}{ Hipertensión } \\
\hline $\mathrm{Si}$ & 112 & 26,1 & 81 & 18,8 & 237 & 55,1 & 430 \\
\hline \multicolumn{8}{|l|}{ Enfermedad hepática } \\
\hline $\mathrm{Si}$ & 1 & 8,3 & 5 & 41,7 & 6 & 50 & 12 \\
\hline \multicolumn{8}{|l|}{ Alcohol } \\
\hline $\mathrm{Si}$ & 7 & 35 & 2 & 10 & 11 & 55 & 20 \\
\hline \multicolumn{8}{|l|}{ Quimioterapia } \\
\hline No & 146 & 27,3 & 108 & 20,2 & 281 & 52,5 & 535 \\
\hline Antraciclinas & 4 & 50 & 2 & 25 & 2 & 25 & 8 \\
\hline Anticuerpos monoclonales & 0 & 0 & 0 & 0 & 2 & 100 & 2 \\
\hline Agentes alquilantes & 1 & 33,3 & 0 & 0 & 2 & 66,7 & 3 \\
\hline Radioterapia & 1 & 50 & 0 & & 1 & 50 & 2 \\
\hline \multicolumn{8}{|l|}{ EPOC } \\
\hline $\mathrm{Si}$ & 26 & 21,5 & 21 & 17,3 & 74 & 61,2 & 121 \\
\hline \multicolumn{8}{|l|}{ Hipertensión pulmonar } \\
\hline $\mathrm{Si}$ & 33 & 27,3 & 21 & 17,3 & 67 & 55,4 & 121 \\
\hline \multicolumn{8}{|l|}{ Infarto } \\
\hline $\mathrm{Si}$ & 59 & 36,2 & 43 & 26,4 & 61 & 37,4 & 163 \\
\hline \multicolumn{8}{|l|}{ Revascularización miocárdica } \\
\hline $\mathrm{Si}$ & 31 & 34,8 & 20 & 22,5 & 38 & 42,7 & 89 \\
\hline \multicolumn{8}{|l|}{ Enfermedad renal crónica } \\
\hline $\mathrm{Si}$ & 39 & 37,5 & 19 & 18,3 & 46 & 44,2 & 104 \\
\hline \multicolumn{8}{|l|}{ Enfermedad vascular periférica } \\
\hline $\mathrm{Si}$ & 15 & 31,2 & 13 & 27,1 & 20 & 41,7 & 48 \\
\hline Enfermedad tiroidea & & & & & & & \\
\hline $\mathrm{Si}$ & 40 & 24,5 & 22 & 13,5 & 101 & 62 & 163 \\
\hline
\end{tabular}


$460(83,6 \%)$ y edema en miembros inferiores en 305 (56\%) pacientes. Según el análisis bivariado, de los pacientes con FEVI preservada (mayor al 50\%), 241 presentaban disnea, y 135 pacientes en el grupo con FEVI disminuida (menor al $40 \%)$.

En cuanto a la etiología de la falla cardiaca, se observa que la causa más prevalente es la isquémica con un $47,8 \%$, seguida por falla cardiaca de origen valvular con $19,3 \%$ y dilatada con 11,8\% (tabla 2). Según la fracción de eyección, se observa que la mayoría de los pacientes con etiología isquémica y valvular se encuentran en el grupo de FEVI preservada $(38,7 \%$ y $72,6 \%)$ (tabla 3$)$. Por otra parte, se evidencia que los pacientes con falla cardiaca de etiología dilatada, se encuentran en un mayor porcentaje $(44,8 \%)$ en el grupo de FEVI reducida.

En el análisis bivariado planteado en la tabla 4, se observa que la mayoría de pacientes con historia de hipertensión arterial, enfermedad coronaria, fibrilación auricular, hipotiroidismo, diabetes, EPOC y enfermedad renal crónica se encuentran en el grupo de FEVI preservada. La mortalidad intrahospitalaria fue de $8,4 \%$, en tanto que la aparición de arritmias se dio en un 22,5\%; al analizarlo por FEVI se observa que la mayoría de pacientes con desenlaces de mortalidad o arritmias se encuentran en el grupo de FEVI preservada.

\section{Discusión}

Las características de los ingresos hospitalarios en pacientes con falla cardíaca aguda carecen de análisis y estudio a conciencia en Colombia. Debido a la importancia y su impacto en salud pública, se creó RECODEC (primera base de datos de enfermedades cardiovasculares), con el objetivo de caracterizar la población de pacientes hospitalizados con falla cardiaca aguda en la fundación Santa Fe de Bogotá. Los datos iniciales del registro proporcionan nuevos conocimientos sobre las características clínicas de estos pacientes y permiten una aproximación más centrada en el paciente al conocer mejor sus condiciones demográficas.

Según los resultados, los pacientes que consultan al hospital por agudización de falla cardiaca comparados con otros registros del país, son por lo general más ancianos (77 años vs. 72,4 años) y en un mayor porcentaje hombres ( $55 \%$ vs. $44,5 \%)^{6}$. En pacientes hospitalizados, el régimen de salud más predominante es medicina prepagada (asistencia privada en salud), ya que este hospital no es una entidad estatal, seguido por afiliación a EPS. Es importante mencionar que esto no es equivalente con el resto de hospitales del país, pues son escasas las instituciones que cuentan con seguridad privada como principal fuente de pacientes.

Al tratarse de una enfermedad crónica, los pacientes a menudo cursan con otras comorbilidades; en este estudio las más significativas fueron hipertensión arterial con 430 pacientes $(78,2 \%)$ y enfermedad coronaria con $211(31,8 \%)$.

En estudios de grandes cohortes de pacientes, alrededor del $66 \%$ de ellos presentan enfermedad coronaria con fracción de eyección disminuida (en este estudio fue más frecuente en el grupo de fracción de eyección preservada) ${ }^{7-9}$; sin embargo, variables como hipertensión arterial y fibrilación auricular tienen como características una fracción de eyección preservada tal y como se evidencia en este estudio ${ }^{5}$.

Los síntomas y signos de consulta más frecuentes, usualmente eran indicativos de congestión pulmonar y sistémica (sensación de disnea y edema de miembros inferiores), lo que es consecuente con aumento de las presiones hidrostáticas en la microcirculación de dichos lechos ${ }^{10}$.

En cuanto a la mortalidad hospitalaria, llama la atención la mayor tasa en el grupo de fracción de eyección preservada, lo cual debe animar a encontrar terapias que tengan efectos sobre desenlaces duros en este conjunto de pacientes, debido a que la mayoría de la evidencia publicada se refiere a fracción de eyección disminuida ${ }^{11-13}$.

Una de las grandes limitaciones del estudio fue la pérdida de datos de algunas variables, que al no encontrarse en las historias clínicas de los pacientes dificultaron su análisis. Para controlar el sesgo de selección y de información, una profesional diferente a los investigadores se encargó de auditar los resultados de RECODEC. No se tuvieron en cuenta las variables con datos perdidos que superaran el $11 \%$ de la población estudiada.

\section{Conclusiones}

Es importante tener en cuenta que el análisis de la base de datos de RECODEC se ha utilizado para evaluar los aspectos epidemiológicos y clínicos de la enfermedad cardiovascular. Los datos aportados, tanto desde el punto de vista administrativo como clínico, han proporcionado información importante acerca del papel en la salud pública y el pronóstico de factores epidemiológicos clásicos, como la edad, el sexo y las comorbilidades en pacientes con falla cardíaca, así como alguna información sobre los desenlaces. En el presente estudio, como desenlaces finales se evaluaron la mortalidad y la aparición de fibrilación auricular, presentes en el $8,4 \%$ y $22,5 \%$ respectivamente; llama la atención la mayor prevalencia de mortalidad en pacientes con FEVI conservada, por lo que se realizarán mas estudios en este grupo de pacientes.

Existe la necesidad de crear registros nacionales que permitan analizar el comportamiento en general de la población colombiana, con el fin de establecer políticas en salud que prevengan el alto porcentaje de morbi-mortalidad en este grupo de pacientes.

\section{Responsabilidades éticas}

Protección de personas y animales. Los autores declaran que para esta investigación no se han realizado experimentos en seres humanos ni en animales.

Confidencialidad de los datos. Los autores declaran que han seguido los protocolos de su centro de trabajo sobre la publicación de datos de pacientes.

Derecho a la privacidad y consentimiento informado. Los autores han obtenido el consentimiento informado de los pacientes y/o sujetos referidos en el artículo. Este documento obra en poder del autor de correspondencia. 


\section{Conflictos de interés}

\section{Ninguno.}

\section{Bibliografía}

1. Instituto nacional de salud, I.N.S. 1. Aspectos relacionados con la frecuencia de uso de los servicios de salud, mortalidad y discapacidad en Colombia, 2011. [Online]. Disponibel en: https://www.minsalud.gov.co/sites/rid/Lists/BibliotecaDigital/ RIDE/IA/INS/informefinal.pdf.[Acceso 10 Dec 2016].

2. Abarquez R, Reganit P, Chungunco C, Alcover J, Punzalan F, Reyes $\mathrm{E}$, et al. Chronic heart failure clinical practice guidelines' class 1-A pharmacologic recommendations: start-to-end synergistic drug therapy? ASEAN Heart J. 2016;24.

3. Lopez A. Assessing the burden of mortality from cardiovascular diseases. World health statistics quarterly Rapport trimestriel de statistiques sanitaires mondiales. 1992;46:91-6.

4. Buitrago AF. Reactions Weekly. 2016;1002:232.

5. Ospina A, Gamarra G. Características clínicas y epidemiológicas de la insuficiencia cardiaca en el Hospital Universitario Ramón Gonzaález Valencia de Bucaramanga, Colombia. Revista Salud UIS. 2004;36:125-31.

6. Hai J, Chan P, Huang D, Ho M, Ho C, Cheung E, et al. Clinical characteristics, management, and outcomes of hospitalized heart failure in a chinese population-The Hong Kong Heart Failure Registry. J Cardiac Failure. 2016;22:600-8.

7. Kociol R, Hammill B, Fonarow G, Klaskala W, Mills R, Hernandez A, et al. Generalizability and longitudinal outcomes of a national heart failure clinical registry: Comparison of Acute Decompensated Heart Failure National Registry (ADHERE) and non-ADHERE Medicare beneficiaries. Am Heart J. 2010;160:885-92.

8. Adams KF Jr, Fonarow GC, Emerman CL, LeJemtel TH, Costanzo MR, Abraham WT, et al. Characteristics and outcomes of patients hospitalized for heart failure in the United States: rationale, design, and preliminary observations from the first 100,000 cases in the Acute Decompensated Heart Failure National Registry (ADHERE). Am Heart J. 2005;149: 209-16.

9. Atherton JJ, Hayward CS, Wan Ahmad WA, Kwok B, Jorge J, Hernandez AF, et al. Patient characteristics from a regional multicenter database of acute decompensated heart failure in Asia Pacific (ADHERE International-Asia Pacific). J Cardiac Fail. 2011;1:82-8.

10. Ponikowski P, Voors A, Anker S, Bueno H, Cleland J, Coats A, et al. 2016 ESC Guidelines for the diagnosis and treatment of acute and chronic heart failure. Eur J Heart Fail. 2016;18:891-975.

11. Martínez-Braña L, Mateo-Mosquera L, Bermúdez-Ramos M, García MD, Hernández ÁV, Ameijeiras LF, et al. Clinical characteristics and prognosis of heart failure in elderly patients. Revista Portuguesa de Cardiologia. 2015;34:457-63.

12. Kajimoto K, Sato N, Takano T. Association between age, time after hospitalization, and survival among patients hospitalized for acute heart failure syndromes. Int J Cardiol. 2016;212:151-3.

13. Martínez-Braña L, Mateo-Mosquera L, Bermúdez-Ramos $M$, García MD, Hernández LF, Ameijeiras ÁH, et al. Clinical characteristics and prognosis of heart failure in elderly patients. Revista Portuguesa de Cardiologia. 2015;34:457-63. 